\title{
TAS WITH QAM-M (M=2, 3, 4, 5, 6) FOR MIMO COMMUNICATION SYSTEM
}

\author{
Hameed Miyan ${ }^{1}$, Kumarikiran ${ }^{2}$ \\ ${ }^{1}$ ECE Department BKIT, Bhalki, VTU, Belgaum \\ ${ }^{2}$ M.Tech Scholar BKIT, Bhalki
}

\begin{abstract}
In this paper we study Orthogonal-Space-Ttime Block-Coded (OSTBC) Multiple-Input Multiple Output (MIMO) systems employing Transmit Antenna Selection (TAS) with frobenius norm as a selection criterion and compares the BER performance for

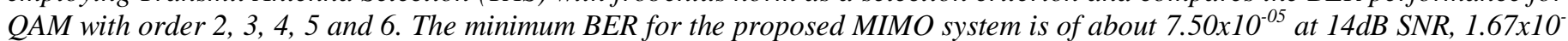

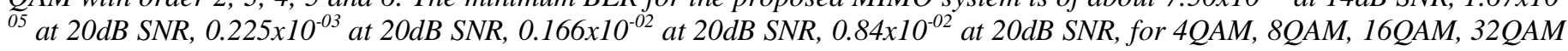
and 64QAM respectively.
\end{abstract}

Keywords: Transmit Antenna Selection (TAS), Multiple Input-Multiple Output, Space Time Block Code (OSTBC), Quaderature Amplitude Modulation (QAM), Bit Error Rate (BER).

\section{INTRODUCTION}

In higher generation technology the capacity of wireless communication will be improved by employing multiple transmitter antennas at the transmitter side and multiple antennas at the receiver side which is termed as multiple input multiple output (MIMO)[1]. The possible gain in performance for a MIMO system is moderated by the increased in the cost as it required separate radio frequency (RF) chain for each antennas which leads to high hardware cost and complexity as well[2]. To reduce the hardware cost and allow the feasibility for MIMO technology a complexity reduction technique can be applied that is known as antenna selection[3]. In antenna selection just a subset of the full array of transmit or/and receive antennas are selected based upon certain criterion. The antennas are attached to the limited number of RF chains by a low cost RF switch which ensures full complexity MIMO technology with smaller number of RF chain[4]. As per the literature survey there exists various antenna selection algorithms which are applied to either transmitter side or at the receive side, or at both transmitter and receiver sides of MIMO systems. A selection criterion in general maximizes the system capacity or diversity[5].

In our previous work we studied an antenna selection algorithms based on a capacity maximization criterion[6-7]. The algorithms used in[6] is iteratively removes antennas from a full set of antennas that results in maximum capacity. Complexity reduced antenna selection algorithm was also studied in[7].After successful study of these algorithms, now in order to obtain the error performance of MIMO systems with antenna selection we had chosen orthogonal space time block coding (OSTBC) is a MIMO transmit action due to its decoding simplicity and high performance[8-9].

In this paper a 2X1 Transmit Antenna Selection(TAS) applied to 4X1 MIMO system and frobenius norm used as a selection criterion and different modulation order with different probability is used to study the BER performance.

\section{SYSTEM MODEL}

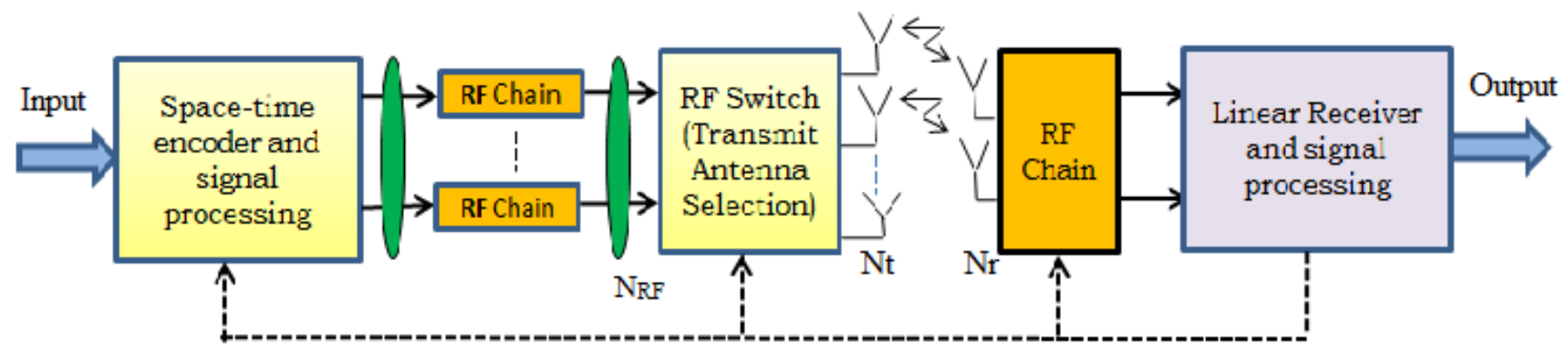

Control and Antenna Selection signaling feedback

Figure 2.1: A STBC system with TAS 
The proposed system model for OSTBC based MIMO system with TAS is shown in Figure 2.1. The generalized equation for received signal is given as,

$Y=H S+Z$

Here the received signal vector is $\mathbf{Y}$, OSTBC encoded signal vector is $\mathbf{S}, \mathbf{H}$ is channel gain matrix and $\mathbf{Z}$ is a noise vector.

\section{A) Performance Analysis}

The error probability for OSTBC is given as,

$P\left(C_{i} \rightarrow C_{j} \mid H_{e f f}\right)=Q\left(\sqrt{\frac{\rho\left\|H_{e f f} E_{i, j}\right\|_{F}^{2}}{2 N_{t}}}\right) \leq$

$\exp \left(-\frac{\rho\left\|H_{e f f} E_{i, j}\right\|_{F}^{2}}{4 N_{t}}\right)$

Where, $C_{i}$ is the transmitted OSTBC code and $C_{j}$ be the encoded for the given error probability of the channel matrix $\mathrm{H}$.

$\mathrm{H}_{\mathrm{eff}}=H_{\left\{P_{1}, P_{2}, \ldots . . P_{Q}\right\}}$ is the effective channel matrix with $\mathrm{Q}$ columns.

TAS is carried out as per the equation 2.3

$\left\{P_{1}^{o p t}, P_{2}^{o p t}, P_{3}^{o p t}, \ldots \ldots P_{Q}^{o p t}\right\}=$

$\arg \max _{P_{1}, P_{2}, P_{3}, . . P_{Q} \in A_{Q}}\left\|H_{e f f}\right\|_{F}^{2}$

Since selected antenna is very much less than the transmit antennas, the average SNR at the receiver side for $Q$ selected antennas $\left\{P_{i}\right\}_{i=1}^{Q}$ is given by,

$(S N R)_{A v g}=\frac{\rho}{Q}\left\|H_{e f f}\right\|_{F}^{2}$

If selected antenna is very much less than number of transmit antennas then equation 2.4 can be rewritten as

$\left\|H_{e f f}\right\|_{F}^{2}=\|H\|_{F}^{2}$

From equation 2.5 the average received SNR is a function of $\|H\|_{F}^{2}$. Which leads to maximum diversity[10].

A) Selection Criterion: Frobenius norm based selection: For a certain number of selected antennas ' $Q$ ' out of ' $\mathrm{N}_{\mathrm{T}}$ ' we can select the antenna subset ' $\mathrm{Lp}$ ' from $\left(\mathrm{N}_{\mathrm{T}} \mathrm{XN}_{\mathrm{R}}\right)$ such that, $L_{p}=\operatorname{avg} \max _{Q \in\left(\mathrm{N}_{\mathrm{T}} \mathrm{XN}_{\mathrm{R}}\right)}\|H\|_{F}^{2}$, to

Minimize the probability error.

B) Modulation Scheme: QAM modulation schemes is used different order, here in each case we had taken phase offset as zero, constellation is [-1 1], symbol order: Gray, symbol mapping:[0 1] and input data type is Bit.

\section{RESULTS AND DISCUSSION}

We had simulated the Antenna selection algorithm applied to 4X1 MIMO systems out of which two transmit antennas are selected for transmission of data. For our simulation we had considered 100 packets and 100 frames and the range of SNR will be 0 to $20 \mathrm{~dB}$. Error performance had being checked for different modulation schemes such as 4QAM, 8QAM, 16QAM,32QAM and 64QAM and it is found that in each cases the BER will be decreases as SNR increases. From the graphs (figure 3(a) to 3(e)) it is observed that the graph is much stripper then the conventional alamouti scheme [12] this shows the better achievement of diversity gain with required minimum number of RF modules this leads to the reduction of hardware cost of MIMO. Also it is found that the minimum BER for the proposed MIMO system is about $7.50 \times 10^{-05}$ at $14 \mathrm{~dB}$ SNR, $1.67 \times 10^{-05}$ at $20 \mathrm{~dB}$ SNR, $0.225 \times 10^{-03}$ at $20 \mathrm{~dB}$ SNR, $0.166 \times 10^{-02}$ at $20 \mathrm{~dB}$ SNR and $0.84 \times 10^{-02}$ at $20 \mathrm{~dB}$ SNR for 4QAM, 8QAM, 16QAM, 32QAM and 64QAM respectively.

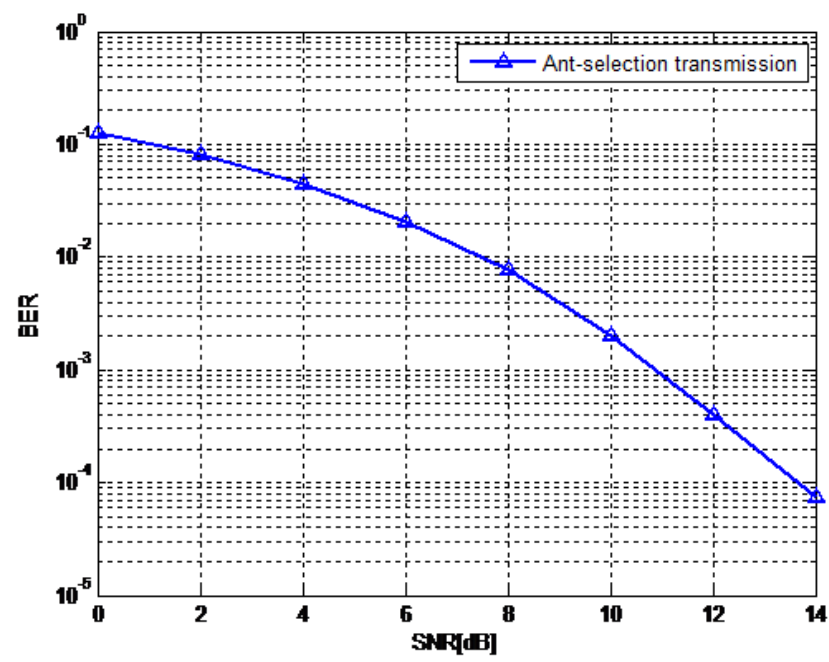

Figure 3(a): 2x1 TAS in 4x1 MIMO with QAM-4

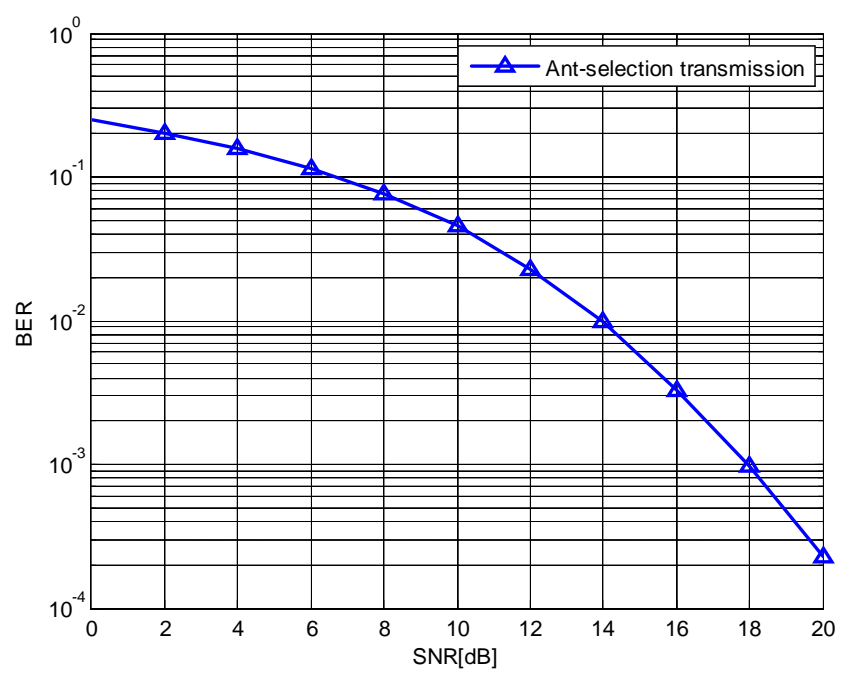

Figure3 (b): 2x1 TAS in 4x1 MIMO with QAM-8 


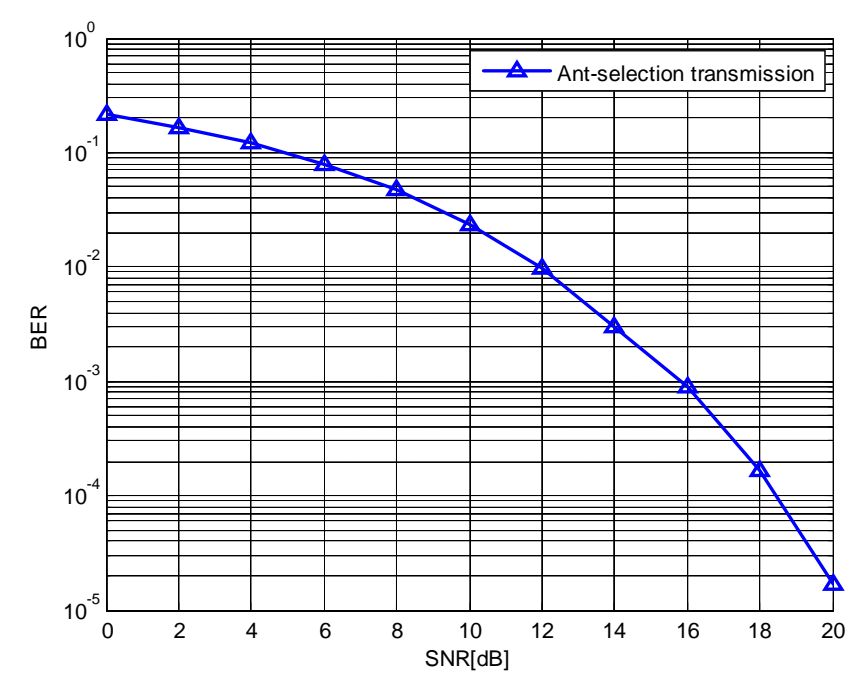

Figure 3(c): 2x1 TAS in 4x1 MIMO with QAM-16

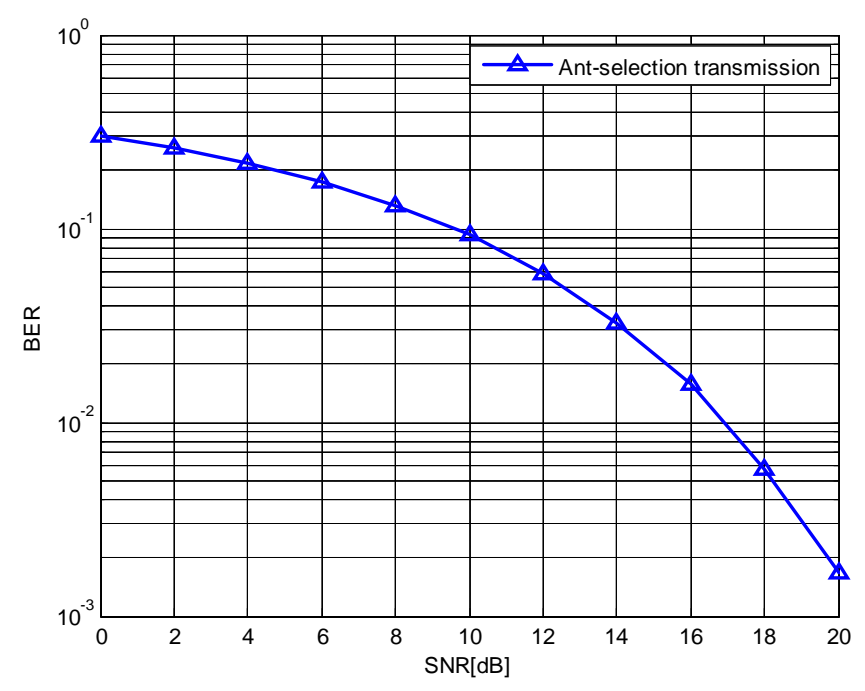

Figure 3(d): 2x1 TAS in 4x1 MIMO with QAM-32

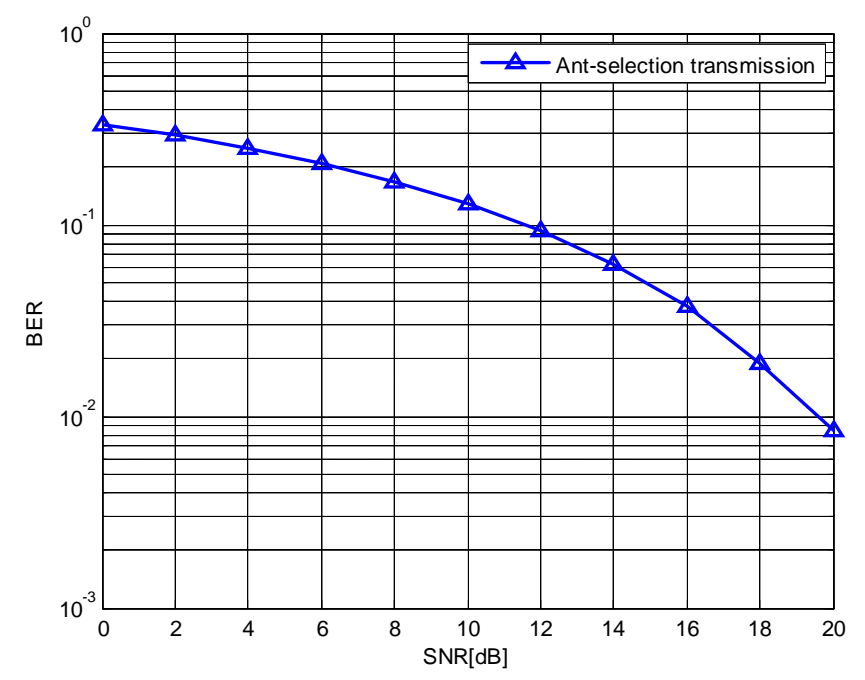

Figure3 (e): 2x1 TAS in 4x1 MIMO with QAM-64

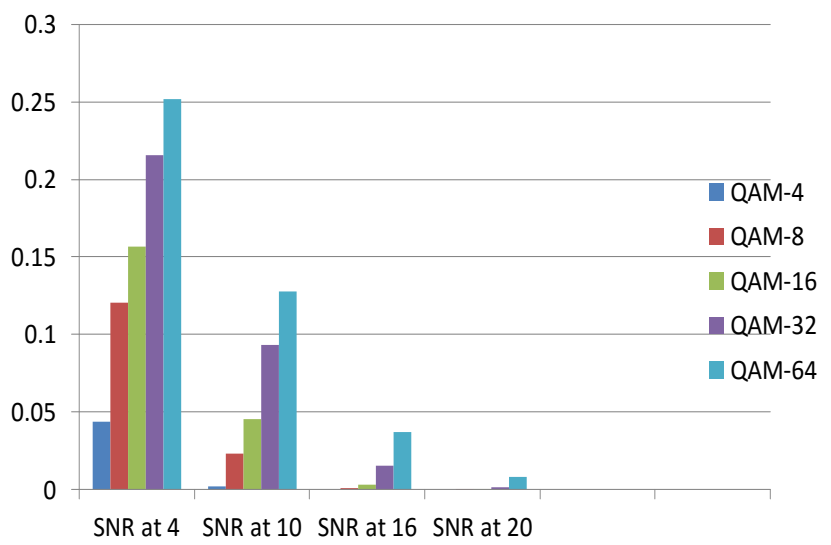

Figure 3.2: Comparison Between Different Modulation techniques Applied to 2x1 SNR

The performance comparison graph of different modulation schemes is shown in figure3.2. In this case the error performance is compared at $4 \mathrm{~dB}, 10 \mathrm{~dB}, 16 \mathrm{~dB}$ SNRs and in all cases it is observed that as order of modulation increases the BER increases this is due to the fact that as the modulation order increases the number of bits per symbol increases for respective modulation scheme and due to the difference in their spatial position as well overlapping leads to the losses in the bits will encounter which is the main cause behind the reduction in BER. Comparison of different modulation schemes in terms of throughput is shown in below table 3. The number of transmitted bits is given by, Simulation bits $=$ Number of bits $*$ Number of Packets and equation and throughput is obtained as,

Throughput $=[($ Transmitted bits - number of bits in error $) /$ Transmitted bits] $* 100 \%$

From the table the best achievable throughput is $99.79 \%$ for QAM-4. And throughput for different modulation order is shown in table 3 .

Table 3: Comparison between Different Modulation Schemes in Terms of the Throughput

\begin{tabular}{|l|l|l|l|}
\hline $\begin{array}{l}\text { Modulation } \\
\text { Scheme }\end{array}$ & $\begin{array}{l}\text { Simulation } \\
\text { Bits }\end{array}$ & $\begin{array}{l}\text { Number of } \\
\text { Bits in } \\
\text { Error }\end{array}$ & $\begin{array}{l}\text { Throughput } \\
(\%)\end{array}$ \\
\hline QAM-4 & 40000 & 81 & $\mathbf{9 9 . 7 9}$ \\
\hline QAM-8 & 60000 & 1385 & 97.69 \\
\hline QAM-16 & 80000 & 3641 & 95.44 \\
\hline QAM-32 & 100000 & 9319 & 90.68 \\
\hline QAM-64 & 120000 & 15318 & 87.23 \\
\hline
\end{tabular}

\section{CONCLUSION}

We had simulated Transmit Antenna Selection technique for different modulation order for QAM-M $(M=2,3,4,5,6)$ in 4X1 MIMO communication system with two selected transmit antennas which leads to reduction of hardware complexity and cost. For the lower modulation order lesser the number of bits in error and better through put. Increasing the modulation order leads to degradation in the 
performance of the system and as well as the degradation of throughput of the system. With OSTBC technique we can achieve better diversity gain, as well as we have seen that using the TAS leads to high speed and low complexity.

\section{ACKNOWLEDGEMENT}

We thankful to Dr.T.S.Vishwanth, Dr.C.M.Tavade for their kind support in developing algorithms, also grateful to Dr.B.B.Lal for providing the research facilities in BKIT, Bhalki.

\section{REFERENCES}

[1]. N.Seshadri and J. Winters, "Two signaling schemes for improving the error performance of frequency division duplex (FDD) transmission systems using transmitter antenna diversity", Int.J.Inform.Networks, vol.1,pp.4960, Jan.1994.

[2]. A.F.Molisch \& M.Z. Win, "MIMO systems with antenna selection", IEEE Microw.Mag.vol.5no.1, pp.46-56, Mar.2004.

[3]. A.F.Molisch, M.Z. Win and J.H.Winters, "Capacity of MIMO systems with antenna selection", in proc. IEEE Int. Conf.Commun. Jan. 2001, vol.2, pp.570-574.

[4]. Suoping Li, Hongfeng and Dandan Xiao, " A low Complexity Algorithms for Receiver Antenna Selection in MIMO Systems", Appl. Math.Inf. Sci., vol.7,no.6, pp.2297-2303, Jul.2013.

[5]. Shahab Sanayei and Aria Nosratinia, "Antenna Selection in MIMO Systems", IEEE Commun.Mag. pp.68-73, Oct. 2004.

[6]. Hameed Miyan, Deepika Patil and S.G.Hiremath, “ Capacity based performance analysis of optimal antenna selection for 8X8 MIMO", IOSR J. of Elec. and Commun. Engg. (IOSR-JECE)vol.10,no.2,pp.0811, Mar- Apr.2015.

[7]. Hameed Miyan and Deepika Patil, "Low Complexity Antenna selection technique for MIMO system", Ind. J. of Appl. Research vol.5,no.5,pp.19-21, May. 2015.

[8]. D. Gore and A. Paulraj, "MIMO antenna subset selection with Space Time Coding”, IEEE Trans. Signal Process.Vol.50, no.10, pp.2580-2588, Oct.2002.

[9]. Dimas Mavares T and Refeal P. Torres," Space-Time Code Selection for Transmit Antenna Diversity systems", IEEE Trans. on Vehic., Tech., vol.57,no.1, Jan. 2008.

[10]. Vaibhav Hendre, M.Murugan and Akshay B. Chandgude, "Multiple Transmit Antenna Selection in MIMO-OSTBC System in Nakagami-m Fading Channel", ScienceDirect, $11^{\text {th }}$ Int.Multi-Conf. on Info. Proc.-2015(IMCIP-2015) Procedia Computer Science 54 pp. $558-565,2015$. 